\title{
Limiting stress states in granular avalanches
}

\author{
Y. G. TAI, J. M. N.T. GraY \\ Institut für Mechanik, Technische Universität Darmstadt, D-64289 Darmstadt, Germany
}

\begin{abstract}
The Savage-Hutter theory for granular avalanches assumes that the granular material is in either of two limiting stress states, depending on whether the motion is convergent or divergent. At transitions between convergent and divergent regions, a jump in stress occurs, which necessarily implies that there is a jump in the avalanche velocity and/or its thickness. In this paper, a regularization scheme is used, which smoothly switches from one stress state to the other, and avoids the generation of such singular surfaces. The resulting algorithm is more stable than previous numerical methods but shocks can still occur during rapid convergence in the run-out zone. Results are presented from two-dimensional calculations on complex geometry which illustrate that some necking features observed in laboratory experiments can be explained by the regularized Savage-Hutter model.
\end{abstract}

\section{INTRODUCTION}

The material properties of dense-flow avalanches, of snow, ice or rocks, are described by a simple Mohr-Coulomb criterion (Savage and Hutter, 1989, 1991). This provides sufficient information to determine the limiting normal pressures within the flowing avalanche but provides no information about the transition between these limiting states or which limiting state is associated with a given deformation. In this paper, a regularization process is introduced which provides smooth well-defined transitions between the various states.

\section{GOVERNING EQUATIONS}

In the extended Savage-Hutter theory for granular freesurface flows over complex topography (e.g. Gray, in press), a slope-fitted curvilinear coordinate system $O x y z$ is generated by a reference surface that follows the "mean" down-slope chute topography. The $x$ and $y$ axes are oriented in the down-slope and cross-slope directions to the reference surface and the $z$ axis is normal to it. The complex shallow three-dimensional chute topography over which the avalanche flows is then defined by its elevation $z=b(x, y)$ above the reference surface.

To leading order, the depth-integrated mass balance reduces to

$$
\frac{\mathrm{d} h}{\mathrm{~d} t}+h\left(\frac{\partial u}{\partial x}+\frac{\partial v}{\partial y}\right)=0
$$

where $h$ is the avalanche thickness, $u$ and $v$ are the depthaveraged velocity components in the down-slope and crossslope directions and the total derivative $\mathrm{d} / \mathrm{d} t=\partial / \partial t$ $+u \partial / \partial x+v \partial / \partial y$. The leading-order depth-integrated momentum balance components are

$$
\begin{aligned}
& \frac{\mathrm{d} u}{\mathrm{~d} t}=a_{x}-g \cos \zeta\left(K_{x} \frac{\partial h}{\partial x}+\frac{\partial b}{\partial x}\right), \\
& \frac{\mathrm{d} v}{\mathrm{~d} t}=a_{y}-g \cos \zeta\left(K_{y} \frac{\partial h}{\partial y}+\frac{\partial b}{\partial y}\right)
\end{aligned}
$$

where $g$ is the gravitational acceleration, $\zeta$ is the local slopehttps://doi.org/10.3189/1998AoG26-1-272-276 Published online by Cambridge University Press 272 inclination angle, $K_{x}$ and $K_{y}$ are the down- and cross-slope earth-pressure coefficients and $\partial b / \partial x$ and $\partial b / \partial y$ are the basal-topography gradients. The gravity-acceleration and basal-drag terms are combined into net driving forces

$$
\begin{aligned}
& a_{x}=g \sin \zeta-(u /|\mathbf{u}|) \tan \delta\left(g \cos \zeta+\kappa u^{2}\right), \\
& a_{y}=\quad-(v /|\mathbf{u}|) \tan \delta\left(g \cos \zeta+\kappa u^{2}\right),
\end{aligned}
$$

in down- and cross-slope directions, respectively, where $|\mathbf{u}|=\left(u^{2}+v^{2}\right)^{\frac{1}{2}}$ is the modulus of the velocity components, $\delta$ is the basal Coulomb dry-friction angle and $\kappa$ is the local curvature of the reference surface.

The earth-pressure coefficients $K_{x}$ and $K_{y}$ relate the limiting in-plane stresses to the normal stress within the avalanche. For avalanches whose motion is predominantly in the down-slope direction, Hutter and others (1993) showed that

$$
\begin{aligned}
& K_{x_{\text {act } / \mathrm{pas}}}=2 \sec ^{2} \phi\left(1 \mp \sqrt{1-\cos ^{2} \phi \sec ^{2} \delta}\right)-1, \\
& K_{y_{\text {act } / \mathrm{pas}}}^{x}=\frac{1}{2}\left(K_{x}+1 \mp \sqrt{\left(K_{x}-1\right)^{2}+4 \tan ^{2} \delta}\right)
\end{aligned}
$$

where $\phi$ is the internal angle of friction in the Mohr-Coulomb criterion. The subscripts act and pas denote active- and passive-stress states, respectively. Greve and others (1994) introduced the following ad hoc definitions to decide which of the stress states should be associated with a given deformation

$$
\begin{aligned}
& K_{x}= \begin{cases}K_{x_{\text {act }}}, & \partial u / \partial x>0, \\
K_{x_{\text {pas }}}, & \partial u / \partial x<0,\end{cases} \\
& K_{y}=\left\{\begin{array}{lll}
K_{y_{\text {act }}}^{x_{\text {act }}}, & \partial u / \partial x>0, & \partial v / \partial y>0, \\
K_{y_{\text {aat }}}^{x_{\text {ans }}}, & \partial u / \partial x<0, & \partial v / \partial y>0, \\
K_{y_{\text {pas }}}^{x_{\text {act }}}, & \partial u / \partial x>0, & \partial v / \partial y<0, \\
K_{y_{\text {pas }}}^{x_{\text {pas }}}, & \partial u / \partial x<0, & \partial v / \partial y<0 .
\end{array}\right.
\end{aligned}
$$

Active-stress states are associated with a dilation of the material, whilst passive-stress states are associated with a compression. In Figure 1, the down- and cross-slope earthpressure coefficients are plotted as functions of the internal angle of friction. 

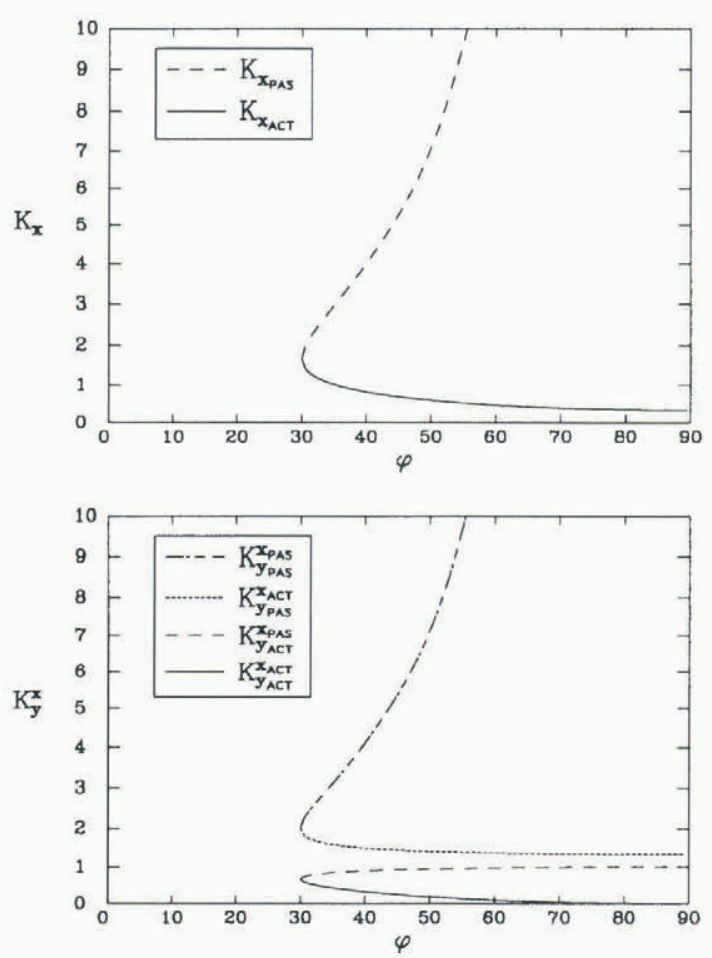

Fig. 1. The down-slope (top panel) and cross-slope (bottom panel) earth-pressure coefficients are plotted as a function of the internal angle of friction $\phi$. The various active and passive states are indicated by differing line styles. Note, that neither $K_{x}$ nor $K_{y}$ are defined for $\phi<\delta=30^{\circ}$

\section{REGULARIZATION OF THE THEORY}

The earth-pressure coefficients jump from active to passive states when either $\partial u / \partial x=0$ or $\partial v / \partial y=0$. It follows that there is a jump in the in-plane stress between convergent and divergent regions and that there must also be a corresponding jump in the avalanche velocity, and/or the thickness, in order to balance the tractions on either side of the interface. Such transitions where the variables jump are called singular surfaces (e.g. Chadwick, 1976).

A proper integration of the Savage-Hutter theory requires the solution of jump conditions on multiple non-material moving boundaries between evolving regions of conver-

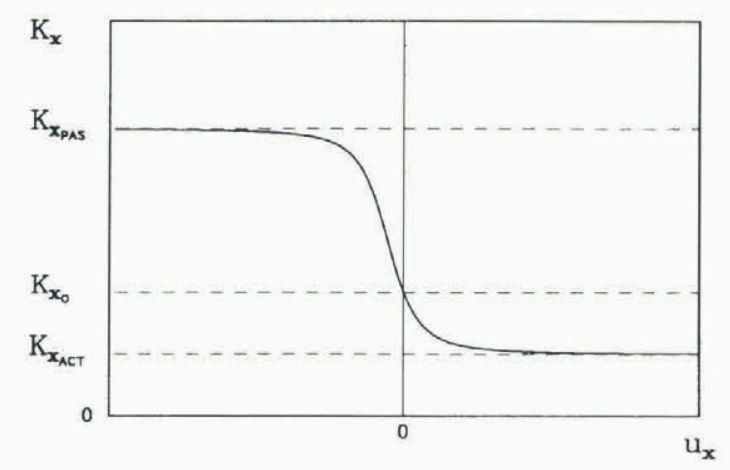

Fig. 2. The down-slope earth pressure is regularized by introducing a smoothly varying monotonically decreasing function of the down-slope divergence $\partial u / \partial x$, which approaches the limiting values, $K_{x_{a c t}}$ and $K_{x_{p a x}}$, for large divergence and convergence, respectively. At $\partial u / \partial x=0$ the down-slope earth pressure equals $K_{x_{0}}$. gent and divergent motion. This is a truly formidable task. An alternative approach is to regularize the theory by introducing a smooth transition between the various limiting stress states. This is illustrated schematically in Figure 2 for the down-slope earth-pressure coefficient. For large downslope convergence $K_{x}$ approaches $K_{x_{p a s}}$ and for large down-slope divergence $K_{x}$ approaches $K_{x_{a c t}}$. Between these two limiting states there is a smooth monotonically decreasing transition, which crosses the $\partial u / \partial x=0$ line at $K_{x}=K_{x_{0}}$.

To formalize the regularization of the Savage-Hutter model, new down- and cross-slope earth-pressure coefficients are introduced

$$
\begin{aligned}
& K_{x}=\left(K_{x_{\text {act }}}+K_{x_{\text {pus }}}\right) / 2+F\left(K_{x_{\text {act }}}-K_{x_{\text {pas }}}\right) / 2, \\
& K_{y}=\left(K_{y_{\text {act }}}+K_{y_{\text {pas }}}\right) / 2+G\left(K_{y_{\text {act }}}-K_{y_{\text {pus }}}\right) / 2
\end{aligned}
$$

where the functions $F$ and $G$ are now dependent on the down- and cross-slope velocity gradients $u_{x}=\partial u / \partial x$ and $v_{y}=\partial v / \partial y$, respectively. The nature of these functions is somewhat subjective. For the purposes of this paper $F$ and $G$ are assumed to be the monotonically decreasing functions

$$
\begin{aligned}
& F=\left(\alpha u_{x}-u_{x_{0}}\right) /\left\{1+\left(\alpha u_{x}-u_{x_{0}}\right)^{2}\right\}^{\frac{1}{2}}, \\
& G=\left(\alpha v_{y}-v_{y_{0}}\right) /\left\{1+\left(\alpha v_{y}-v_{y_{0}}\right)^{2}\right\}^{\frac{1}{2}}
\end{aligned}
$$

where the parameter $\alpha$ determines the steepness of the transition. The constants $u_{x_{0}}$ and $v_{y_{0}}$ are chosen so that at the origin $\left.K_{x}\right|_{u_{x}=0}=K_{x_{0}}$ and $\left.K_{y}\right|_{v_{y}=0}=K_{y_{0}}$, which implies that

$$
\begin{aligned}
& u_{x_{0}}=-F_{0} /\left(1-F_{0}^{2}\right)^{1 / 2}, \\
& v_{y_{0}}=-G_{0} /\left(1-G_{0}^{2}\right)^{1 / 2}
\end{aligned}
$$

where

$$
\begin{aligned}
& F_{0}=\left(2 K_{x_{0}}-K_{x_{\text {act }}}-K_{x_{\text {pas }}}\right) /\left(K_{x_{\text {act }}}-K_{x_{\text {pas }}}\right), \\
& G_{0}=\left(2 K_{y_{0}}-K_{y_{\text {act }}}-K_{y_{\mathrm{pas}}}\right) /\left(K_{y_{\text {act }}}-K_{y_{\mathrm{pus}}}\right) .
\end{aligned}
$$

The values at the origin are an important feature of the regularized Savage-Hutter model. A natural partitioning of the active- and passive-stress states is achieved when the down- and cross-slope earth-pressure coefficients cross the origin at

$$
K_{x}^{\text {nat }}=2 \sec ^{2} \phi-1, \quad K_{y}^{\text {nat }}=1,
$$

since $K_{x_{\text {act }}} \leq K_{x}^{\text {nat }} \leq K_{x_{\text {pas }}}$ and $K_{y_{\text {act }}} \leq K_{y}^{\text {nat }} \leq K_{y_{\text {pax }}}$ for all values of the internal and basal friction angles. In general, other models are possible and three situations are considered here

$$
\begin{aligned}
& \text { (1). } K_{x_{0}}=K_{x}^{\text {nat }}, \quad K_{y_{0}}=K_{y}^{\text {nat }} \text {, } \\
& \text { (2). } \quad K_{x_{0}}=K_{x_{\text {act }}}+\varepsilon, \quad K_{y_{0}}=K_{y_{\mathrm{act}}}+\varepsilon, \\
& \text { (3). } \quad K_{x_{0}}=K_{x_{\text {pas }}}-\varepsilon, \quad K_{y_{0}}=K_{y_{\text {art }}}-\varepsilon
\end{aligned}
$$

where $\varepsilon$ is a small parameter that is introduced to ensure that the transition occurs close to the origin. The first of these models shall be referred to the naturally regularized model, the second as the active model and the third as the passive model.

\section{NUMERICAL GONSIDERATIONS}

It is appropriate at this point to discuss briefly some of the finer points involved in a numerical algorithm to solve the two-dimensional regularized Savage-Hutter theory. In many respects, the algorithm adopted here is similar to that used in existing Lagrangian schemes to solve the standard 
Savage -Hutter model (e.g. Koch and others, 1994). The important difference arises in the way in which the earthpressure coefficients at a gridpoint are calculated from the velocity gradients at a given time-step.

In existing schemes, the velocity gradients are computed for a triangular gridcell and the appropriate earth-pressure values for that gridcell are determined from relations (6) and (7). Once this has been performed for all gridcells the earth-pressure coefficients at a gridpoint are determined by volumetric gridcell averages

$$
\begin{aligned}
& \left(K_{x}\right)_{\text {point }}=\frac{\sum\left(K_{x}\right)_{\text {cell }} V_{\text {cell }}}{\sum V_{\text {cell }}}, \\
& \left(K_{y}\right)_{\text {point }}=\frac{\sum\left(K_{y}\right)_{\text {cell }} V_{\text {cell }}}{\sum V_{\text {cell }}}
\end{aligned}
$$

where the summations are performed over the set of adjacent gridcells to the point and $V_{\text {cell }}$ is the volume of the gridcell. At gridpoints that lie close to singular surfaces, this scheme has the property that the volumetric mean earth pressure lies between the active and passive values. It follows that the earth-pressure coefficients are implicitly smoothed by the algorithm.

In the algorithm proposed here, the order of the operations is interchanged. First the velocity gradients $\left(u_{x}\right)_{\text {point }}$ and $\left(v_{y}\right)_{\text {point }}$ are calculated by a volumetric average of $\left(u_{x}\right)_{\text {cell }}$ and $\left(v_{y}\right)_{\text {cell }}$ at adjacent gridcells and then the earthpressure coefficients $\left(K_{x}\right)_{\text {point }}$ and $\left(K_{y}\right)_{\text {point }}$ are calculated directly. It follows that there is no implicit smoothing of the earth-pressure coefficients near a singular surface and this method is therefore appropriate for the regularized model.

\section{EXPERIMENTAL COMPARISON}

To test the regularized Savage-Hutter theory and the new numerical method, a comparison of the results is made with a carefully controlled laboratory experiment, which has already been successfully modelled using the standard method (Gray, 1997).

The chute geometry consists of a channel, inclined at $\zeta=40^{\circ}$ to the horizontal, which is connected to a horizontal plane run-out zone by a smooth transition. This is illustrated in Figure 3. The channel profile is parabolic with a radius of

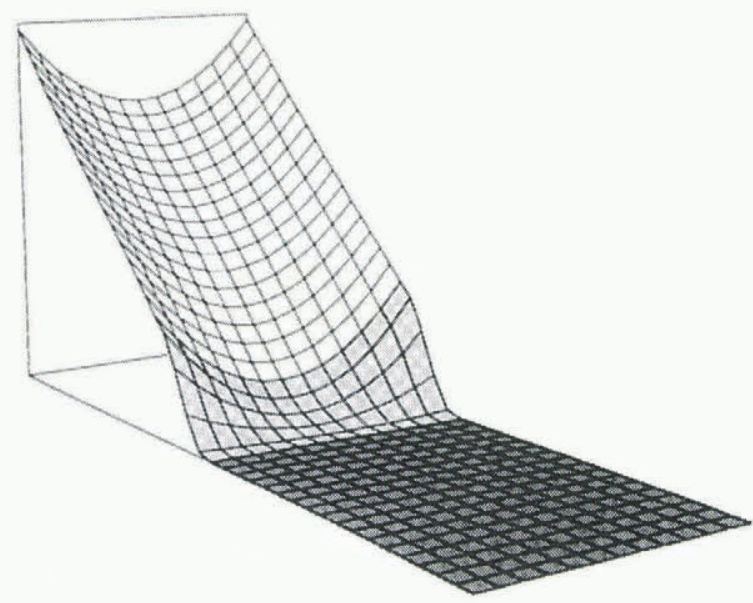

Fig. 3. The basal-chute geometry consists of an the inclined section (white) with shallow parabolic cross-slope profile, which opens out on to a horizontal plane (dark grey). A smooth transition region (light grey) connects the two zones. curvature of $110 \mathrm{~cm}$ and the avalanche is released from a cap that has a spherical free surface and which is fitted to the basal chute topography. The cap radius is $32 \mathrm{~cm}$ and the maximum height of the cap above the free surface is $22 \mathrm{~cm}$.

The experimental data in this paper are taken from experiment V02 (e.g. Gray, 1997), which used quartz granules (mean diameter $2-4 \mathrm{~mm}$ ) that are characterized by an internal angle of friction $\phi=40^{\circ}$ and a basal angle of friction $\delta=27-30^{\circ}$. In numerical results presented in this paper, $\delta$ is assumed to equal $28^{\circ}$ at the front of the avalanche and linear bed-friction angle reduction (Gray, 1997) is used to obtain the correct tail speed.

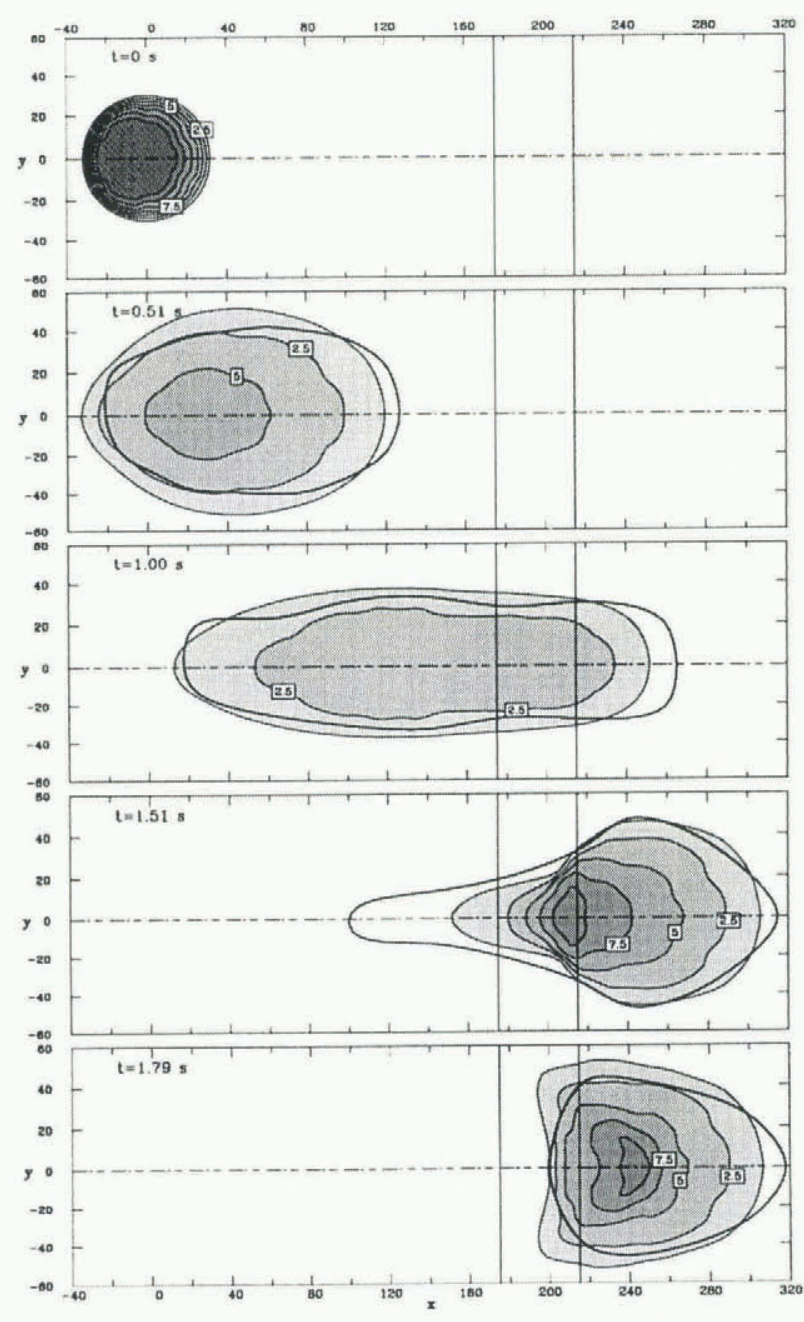

Fig. 4. Avalanche-thickness contours computed using the regularized Savage-Hutter model are plotted using slope fitted curvilinear coordinates at a sequence of time-steps and the thick line shows the position of the experimental avalanche boundary. The time is shown in seconds in each plot and all sizes are in centimetres. The solid lines at $x=175$ and $215 \mathrm{~cm}$ indicate the position of the transition zone and the $y=0 \mathrm{~cm}$ line corresponds to the centre of the channel. The avalanche moves down-slope from left to right.

The predicted avalanche thickness and a comparison with the experimental avalanche boundary is shown at a sequence of time-steps in Figure 4. The avalanche moves down-slope from left to right, starting on the inclined channel in the top panel and coming to rest on the flat run-out plane in the bottom panel. Initially, there is a strong crossand down-slope expansion of the avalanche as the cap is raised but the cross-slope spreading is rapidly balanced by 
the cross-slope topography gradients in the channel and spreading continues predominantly in the down-slope direction. As the avalanche enters on to the run-out plane, the lateral confinement ceases and the avalanche develops a characteristic tadpole form at $t=1.51 \mathrm{~s}$, before coming to rest at $t=1.79 \mathrm{~s}$. At each time-step, the predicted and observed boundaries are in very good agreement, confirming that the regularized Savage-Hutter theory is at least as good if not better than the standard model.

In the laboratory experiment V02, a constriction or necking of the avalanche is observed in the transition zone at $t=1.21 \mathrm{~s}$, as shown in Figure 5 . That is, the maximum width of the avalanche is smaller in the transition zone than in either the channel section or run-out plane. The reason for this behaviour is due to the complex interplay between the chute geometry and material properties of the avalanche as it moves from the channelized to unconfined flow regimes.

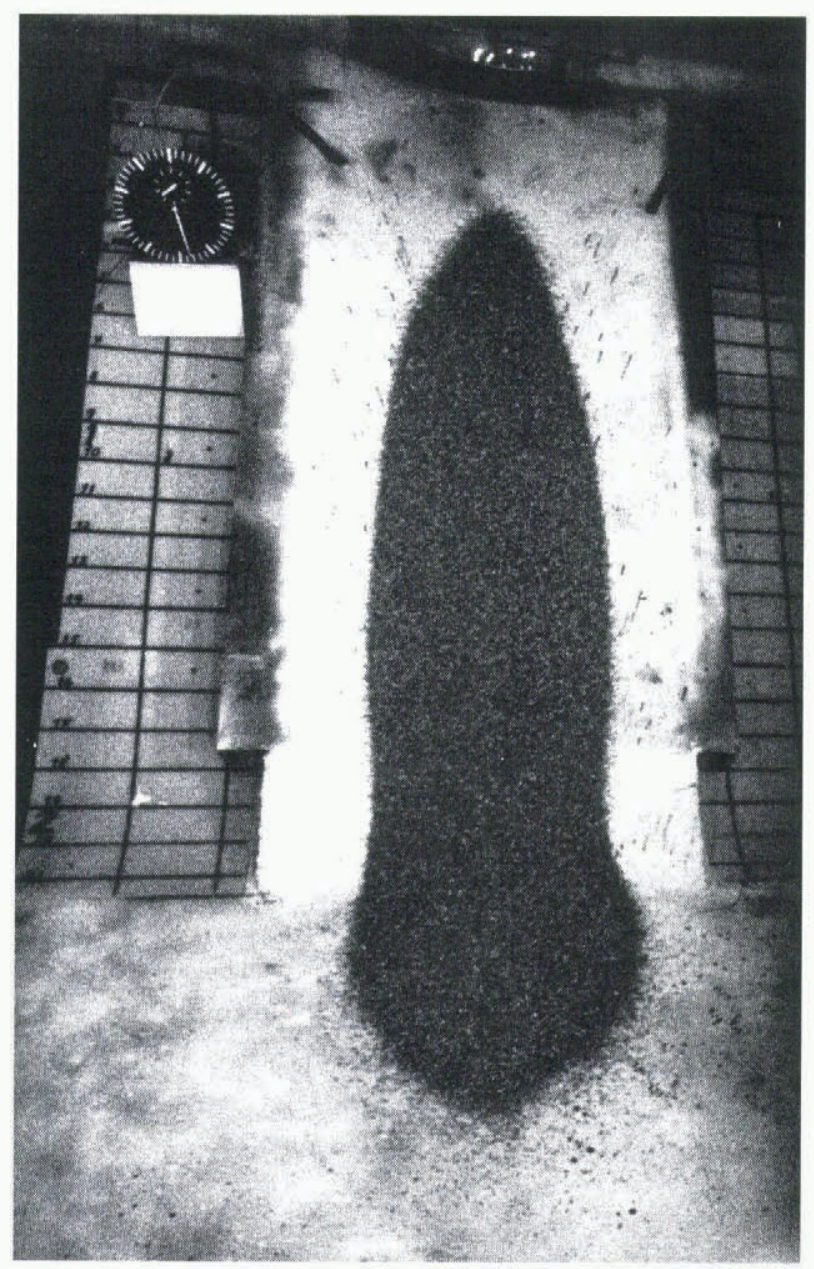

Fig. 5. Necking of the avalanche as observed in experiment V02.

The necking problem provides an interesting test case for both the theory and the numerical methods. Four cases are considered here. The first model is the standard SavageHutter theory using the original numerical method, the second is the naturally regularized Savage-Hutter theory and the third and fourth models illustrate what happens with the standard Savage-Hutter theory when the new numerical method is used. It should be noted that in the ad hoc earth-pressure coefficient definitions, Equations (6) and (7), the cases when either $u_{x}=0$ or $v_{y}=0$, are not defined. Two possibilities are to define

$$
\begin{array}{lll}
\text { (a) } \quad K_{x}=K_{x_{\text {act }}}, & u_{x}=0, \\
K_{y}=K_{y_{\text {act }}}, & v_{y}=0, \\
\text { (b) } \quad & K_{x}=K_{x_{\mathrm{pas}}}, & u_{x}=0, \\
K_{y}=K_{y_{\mathrm{pas}}}, & v_{y}=0 .
\end{array}
$$

The first of these cases is asymptotically similar to the active regularized model for large $\alpha$ and small $\varepsilon$, and the second is asymptotically similar to the passive regularized model, both of which are defined in Equations (13). These models shall therefore be termed the active-jump and passive-jump models, respectively.

In Figure 6 the results of the standard, naturally regularised, active-jump and passive-jump models are illustrated, at time $t=1.21 \mathrm{~s}$ when necking in the transition zone is observed. There is no sign of a constriction in the transition zone with the standard Savage-Hutter model (top panel) but the naturally regularized theory is able to reproduce this phenomenon (upper middle panel). This provides further evidence that the regularization of the earth-pressure coefficients is at least qualitatively correct.

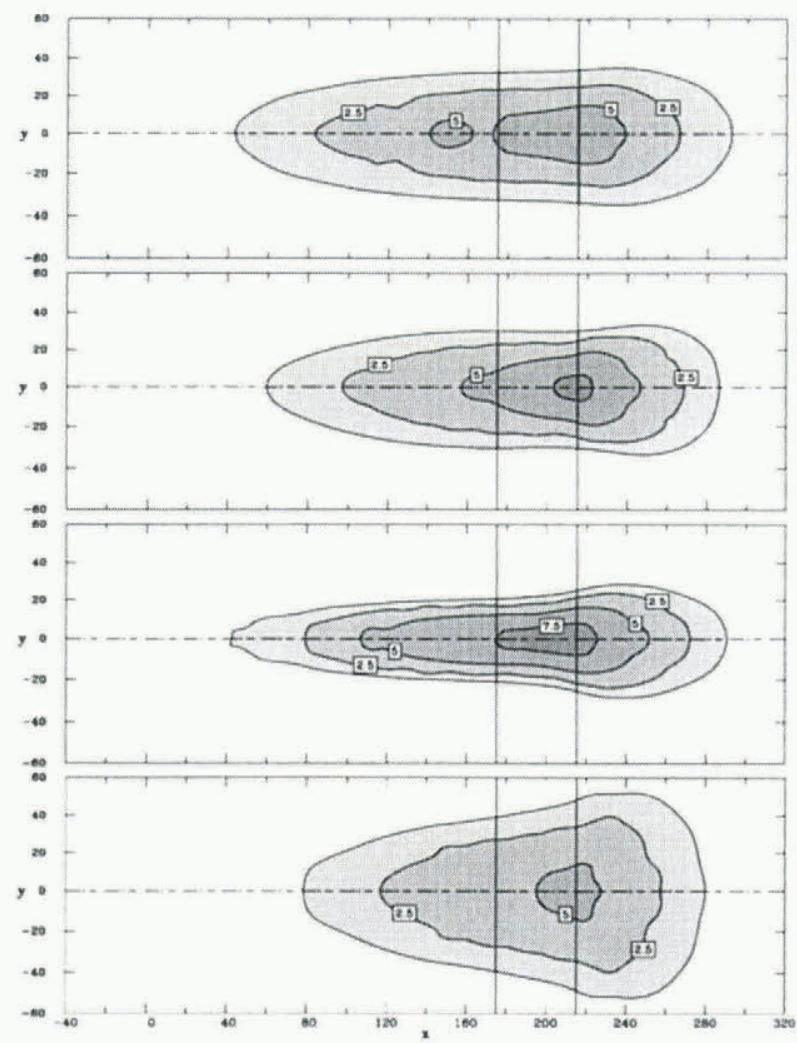

Fig. 6. A sequence of four avalanche-thickness contours are shown for the standard model (top), the naturally regularized model (upper middle), the active-jump model (lower middle) and the passive-jump model (bottom) at $t=1.21 \mathrm{~s}$.

The standard Savage-Hutter model results were produced using the active-jump model (Equations (16)) and the old numerical method. When the passive-jump model is used instead, the results are almost identical. However, when the new numerical method is used, the results for the active-jump model (lower middle panel) are completely different to those obtained with the passive-jump model 
(bottom panel). Indeed, the results for the passive-jump case are similar to experiments performed on unconfined chutes (Koch and others, 1994), where there is no cross-slope curvature. The reason for this is that for a large part of the avalanche motion the cross-slope basal-topography gradients are almost in exact balance with the cross-slope spreading terms in Equations (2) and that the cross-slope velocity and velocity gradients are zero to double precision accuracy. As a result

$$
K_{y}^{\mathrm{a}} \frac{\partial h^{\mathrm{a}}}{\partial y} \simeq \frac{\partial b}{\partial y}, \quad K_{y}^{p} \frac{\partial h^{\mathrm{p}}}{\partial y} \simeq \frac{\partial b}{\partial y},
$$

where the superscript a and p are used to denote activejump and passive-jump models, respectively. It follows from this that

$$
K_{y}^{\mathrm{a}} \frac{\partial h^{\mathrm{a}}}{\partial y} \simeq K_{y}^{\mathrm{p}} \frac{\partial h^{\mathrm{p}}}{\partial y},
$$

implying that the horizontal thickness gradients in the active-jump model are larger than those in the passive-jump model, since $K_{y}^{\mathrm{a}}<K_{y}^{\mathrm{p}}$. The active-jump model therefore produces a much narrower avalanche than the passive-jump model in the channelized section of the chute. These results demonstrate the danger of using a jump function in numerical methods that implicitly assumes the existence of smooth differentiable solutions, as small changes to the nature of the jump can have a large effect on the solution.

\section{CONGLUSIONS}

The regularized Savage-Hutter theory provides a welldefined method of switching from one limiting stress state to another during the avalanche deformation and eliminates the jumps present in the original theory. A comparison of the model results with a carefully controlled laboratory experiment (V02) confirms the superiority of the method over previous schemes.

\section{ACKNOWLEDGEMENTS}

This research was supported by the DFG project SFB 298 "Deformation und Versagen bei metallischen und granularen Strukturen".

\section{REFERENCES}

Chadwick, P. 1976. Continuum mechanics: concise theory and problems. Norwich, George Allen and Unwin Ltd.

Gray, J. M. N.T. 1997. Granular avalanches on complex topography. In Fleck, N., ed. IUTAM Symposium on Mechanics of Granular and Porous Materials, Cambridge. Proceedings. Dordrecht, etc., Kluwer Academic Press, 275-286.

Greve, R., T. Koch and K. Hutter. 1994. Unconfined flow of granular avalanches along a partly curved surface. 1. Theory. Proc. R. Soc. London, Ser. A, 445(1924), 399-413.

Hutter, K., M. Siegel, S. B. Savage and Y. Nohguchi. 1993. Two-dimensional spreading of a granular avalanche down an inclined plane. Part 1 . Theory. Acta Mech., $100(1-2), 37-68$.

Koch, T., R. Greve and K. Hutter. 1994. Unconfined flow of granular avalanches along a partly curved surface. 2. Experiments and numerical computations. Proc. R. Soc. London, Ser. A, 445(1924), 415-435.

Savage, S. B. and K. Hutter. 1989. The motion of a finite mass of granular material down a rough incline. 7. Fluid Mech., 199, 177-215.

Savage, S. B. and K. Hutter. 1991. The dynamics of avalanches of granular materials from initiation to run-out. Part I. Analysis. Acta Mech., 86(1-4), 201-223. 